\title{
The Necessity of Mobile Phone Technologies for Public Health Surveillance in Benin
}

\author{
Yaovi M. G. Hounmanou, ${ }^{1,2,3}$ Murielle S. S. Agonsanou, ${ }^{1}$ \\ Victorien Dougnon, ${ }^{2}$ Mahougnon H. B. Vodougnon, ${ }^{1}$ Ephraim M. Achoh, ${ }^{1}$ \\ Jibril Mohammed, ${ }^{3}$ and Esron D. Karimuribo ${ }^{3}$ \\ ${ }^{1}$ Department of Animal Health and Production, Polytechnic School of Abomey-Calavi, University of Abomey-Calavi, \\ 01 BP 2009 Cotonou, Benin \\ ${ }^{2}$ Research Laboratory in Applied Biology, Polytechnic School of Abomey-Calavi, University of Abomey-Calavi, \\ 01 BP 2009 Cotonou, Benin \\ ${ }^{3}$ Department of Veterinary Medicine and Public Health, Sokoine University of Agriculture, P.O. Box 3121, Chuo Kikuu, \\ Morogoro, Tanzania
}

Correspondence should be addressed to Yaovi M. G. Hounmanou; gilmahu@yahoo.fr

Received 7 June 2016; Revised 19 September 2016; Accepted 29 September 2016

Academic Editor: Jennifer L. Freeman

Copyright (C) 2016 Yaovi M. G. Hounmanou et al. This is an open access article distributed under the Creative Commons Attribution License, which permits unrestricted use, distribution, and reproduction in any medium, provided the original work is properly cited.

\begin{abstract}
A cross-sectional study was conducted in March 2016 to assess the need of mobile phone technologies for health surveillance and interventions in Benin. Questionnaires were administered to 130 individuals comprising 25 medical professionals, 33 veterinarians, and 72 respondents from the public. All respondents possess cell phones and $75 \%, 84 \%$, and $100 \%$ of the public, medical professionals, and veterinarians, respectively, generally use them for medical purposes. $75 \%$ of respondents including $68 \%$ of medics, $84.8 \%$ of veterinarians, and $72.2 \%$ of the public acknowledged that the current surveillance systems are ineffective and do not capture and share real-time information. More than $92 \%$ of the all respondents confirmed that mobile phones have the potential to improve health surveillance in the country. All respondents reported adhering to a nascent project of mobile phonebased health surveillance and confirmed that there is no existing similar approach in the country. The most preferred methods by all respondents for effective implementation of such platform are phone calls (96.92\%) followed by SMS (49.23\%) and smart phone digital forms (41.53\%). This study revealed urgent needs of mobile phone technologies for health surveillance and interventions in Benin for real-time surveillance and efficient disease prevention.
\end{abstract}

\section{Introduction}

The emergence of novel infectious disease threats in both ani$\mathrm{mal}$ and human populations has increased in the world and raising the need for new surveillance capabilities. Although no cases were declared in Benin, the country was recently threatened by Ebola virus disease from its neighbouring countries. However, Benin currently experiences some sporadic cases of Lassa fever, one of the deadliest emerging infectious diseases in the world [1]. Moreover, zoonotic origin of these diseases calls for collaborative efforts and efficient coordination of human and animal health professionals for nascent surveillance systems [2]. Furthermore, the animal population of Benin is exposed to a number of infectious diseases such as tick-borne disease which is currently the leading cause of losses in the livestock sector [3]. It is therefore obvious that the control of infectious diseases poses a major challenge in Benin where the efficiency of health surveillance systems has become limited, hence the need to adopt comprehensive and timely disease surveillance and response systems. This is further backed by the revised International Health Regulations of WHO which requires proactive establishment of infectious disease surveillance system in each member country where surveillance network should be set up [4]. 
In spite of the existence of various regional networks for disease surveillance like the West African Health Organisation (WAHO) to which Benin is a member, the problem of timely reporting still remains rampant [5]. As a paper based reporting system, the essence of these delays is associated with a number of challenges which include poor infrastructures, remoteness of the healthcare facilities from the areas where reports have to be submitted, cost of transportation, and lack of adequate personnel as well as weather conditions $[6,7]$. Additionally, this system demands data reentry at data processing and analysis points, thus making it prone to introduction of errors during this process; all these challenges contribute to delayed acquisition, processing, and response to disease occurrences in remote areas [7]. Currently, of the most promising alternate surveillance systems in the world are those based on mobile technologies. A review on mobile phone-based approaches for health surveillance has led to better reporting systems in order to create community awareness to help build evidence-based field practice in health surveillance in the sub-Saharan Africa [8]. The invention and usage of mobile phones have significantly improved communication and there is a growing global trend in harnessing this technology for behaviour change, disease surveillance, prevention, and control [9]. With a national coverage estimated in June 2015 at $85.94 \%$ of the population, cell phones have become ubiquitous in every corner in Benin [10]. Despite the expansion and increased use of mobile phones for various health interventions around the globe including several African countries [11, 12] and considering the persuasive need to use such technology to improve human and animal health in the country, mobile phones seem to have not yet been introduced into the health surveillance systems in Benin. The current study aimed to investigate the need of mobile phone technologies application for animal and human health surveillance and intervention systems in Benin.

\section{Materials and Methods}

2.1. Materials. Structured pretested questionnaires were designed in French language and administered to human and animal health professionals and the public. These questionnaires were used to probe information including (1) mobile phone ownership, (2) use of mobile phone for health interventions, (3) need of mobile phone in health surveillance, (4) willingness to use mobile phone for health surveillance, and (5) the existence of mobile phone-based health surveillance systems in the country.

\subsection{Methods}

2.2.1. Study Area. The study was conducted in two urban areas Cotonou and Abomey-Calavi and the rural area of Kpomassè Municipality. Cotonou is the largest commercial and administrative city of Benin with high human population and great human contacts, hence posing the risks of disease occurrence and spread. Similar reasons justify the choice of Abomey-Calavi city which is the most populated one of the country. However, Kpomassè was selected for comparison purposes since it is the least developed municipality in Benin. It is a rural municipality where hunting and animal husbandry are the major economic activities making the risk of occurrence and spread of zoonotic diseases high. The three areas are therefore hotspots for health challenges where adequate and appropriate surveillance measures need to be taken. Information coming from these areas could easily be extrapolated to the emergency situation of the entire country, though they are all found in the south.

2.2.2. Study Design and Sample Size. This was a crosssectional study focused on the assessment of the need of mobile phones in health surveillance and interventions in Benin. Conducted from 1st to 15th of March, 2016, study enrolled a total of 25 medical practitioners, 33 veterinarians, and 72 individuals from the general population. The sample size (130) was based on the following inclusion criteria: healthcare centre/veterinary clinic or households located within the study area (Cotonou, Abomey-Calavi and Kpomassè Municipalities); the respondent gave an oral consent to participate in the interview; the respondent was found at the household or the hospital centre/veterinary clinic during the study period; interview was conducted only when all the aforementioned conditions were met.

2.2.3. Sampling Procedure. With respect to the study objective, the method used to select respondents from the public was a systematic random sampling. The rationale is that the study needed to give equal chance to all respondents so as to get comprehensive information from human and animal health perspective and also to avoid bias in the results' interpretation. Therefore, based on information provided by local livestock officers streets with high animal husbandry potentials were identified and every third household in the streets was randomly selected. Questionnaires were administered to one individual per household. Although questionnaires were written in French, the interviews were conducted in French and "Fon" language (local ethnic language) according to respondents' preference. Human and animal healthcare professionals were enrolled to participate in the study based on their availability and presence in their visited offices. For their case, all accessible healthcare centres and veterinary clinics of the study area were visited and only those available during the study period and who complied with the aforementioned inclusion criteria were interviewed.

2.3. Data Analysis. Data were analysed using SPSS version 20. Descriptive statistics was used to analyse the proportion of respondents in specific parameter. Some key proportions such as preferred communication methods were compared between the three different groups and between locations (rural and urban) using chi-square test. Statistical differences were judged at $p<0.05$. Results were presented in tables and graphs constructed in Microsoft Excel based on proportions generated by SPSS. 


\section{Results}

3.1. Possession, Use, and Need of Mobile Phones Technologies in Health Surveillance in Benin. A total of 25 medical practitioners, 33 veterinarians, and 72 individuals in the general population were interviewed in this study. At the end of the study, all 130 respondents possess at least one cell phone (100\%). The majority of respondents $(75 \%, 84 \%$, and $100 \%$ of general population, medical practitioners, and veterinarians, resp.) frequently use their mobile phones for medical purposes. The most common medical activities in which respondents utilise cell phones are for appointments, reporting emergencies, complaints, and follow-ups on medical services/treatments and so forth. Table 1(a) shows more details on these activities and their proportions. Moreover, the common communication methods used by the respondents are phone calls (100\%) followed by short text messages (SMS, $78.46 \%)$ and the least by internet applications like WhatsApp (52.31\%, Table $1(\mathrm{a}))$.

About $92 \%$ of all respondents composed of $88 \%$ of medical practitioner, $91.7 \%$ of respondents from the general public, and $97 \%$ of veterinarians agreed that mobile phones can improve health interventions and surveillance. Moreover, three-quarters of respondents (68\% of medics, $72.2 \%$ individuals from the public, and $84.8 \%$ of veterinarians) confirmed that the current disease reporting and surveillance systems used in the country are ineffective in disseminating and do not capture and share real-time information.

The most common means currently used in Benin for disease reporting are the television, newspapers, radios, and social media and through colleagues mainly by professional associations (Table 1(b)). However, all interviewed individuals $(100 \%)$ regardless of their profession and location affirmed being ready for the use of mobile phones for health interventions and surveillance. Furthermore, respondents listed a number of issues that could be reported using mobile phone-based surveillance platforms. These include outbreaks reporting, cases reporting, follow-ups and controls, appointments, vaccination campaigns, drug reminders (including ARVs), and emergencies (Table 1(b)).

Furthermore, none of the 130 respondents have declared the existence of any mobile phone-based surveillance system in the country.

3.2. Preferred Methods for Effective Mobile Phone-Based Surveillance Platform. For an effective implementation of mobile phone-based health surveillance in Benin, respondents prefer phone calls more than SMS and smart phone digital forms $(96.92 \%, 49.23 \%$, and $41.53 \%$ for calls, SMS, and digital forms, resp.). These were supported by statistical significance $(p<0.05$, Figure 1$)$.

Further statistical comparisons revealed that individuals from the public are less likely to use SMS and digital forms than health professionals $(p=0.014$ for SMS and $p=0.007$ for digitals; Table 1(a)). Nevertheless, the preferences were not statistically affected by the location (urban or rural) of respondents $(p>0.05$, Table $1(\mathrm{a}))$.

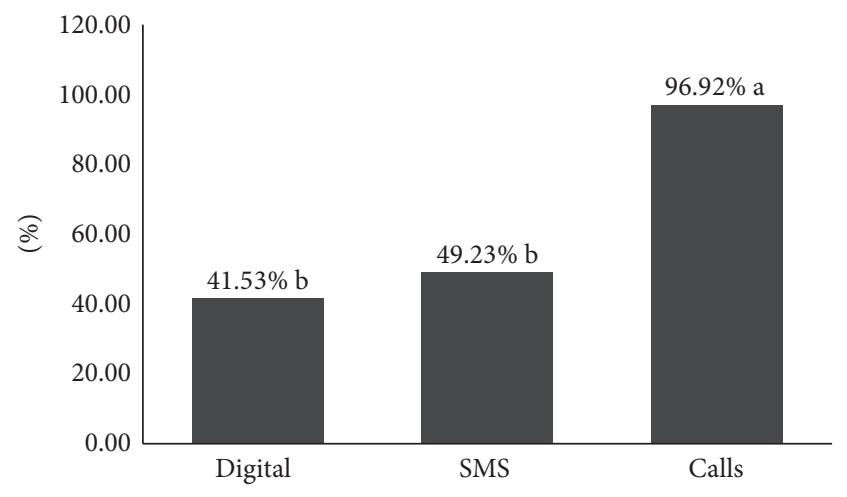

Figure 1: Comparison of preferred reporting methods. Proportions followed by different letters are significantly different $(p<0.05)$.

\section{Discussion}

This study assessed the need of mobile phone technologies for animal and human health surveillance and interventions in Benin and revealed that all respondents own mobile phones. The high level of cell phone possession among respondents is certainly due to the increased national cell phone coverage estimated in June 2015 at $85.94 \%$ in Benin [10] and a teledensity of about $83.81 \%$ in 2014 [13]. People not only own cell phones but also use them for medical and veterinary purposes. These are predisposing conditions that could facilitate the integration of mobile phone-based activities in the country. Furthermore, the fact that respondents confirmed that the current surveillance systems in the country are not real time and also ineffective is evident enough to call for the adoption of alternate innovative surveillance systems leading to timely and effective health surveillance as previously reported by Brinkel et al. [8]. Moreover, findings of this study suggest that a mobile phone-based surveillance system can easily be adopted and implemented in Benin since all the respondents expressed their willingness to use such platform. This positive response could be due to the absence of a similar project in the country in addition to the ineffective surveillance system for disease control. Also healthcare issues including disease outbreaks or other health emergencies and vaccinations, which respondents confirmed as reportable using mobile phone platforms, show that health surveillance and other health interventions can be carried out with this system. This is a great opportunity but unfortunately still untapped potential of mobile phone usage in Benin. There are five telecommunication providers in the mobile phone sector in Benin including four private operators (MTN, MOOV, GLO, and BBCOM) and one public (LIBERCOM) [10]. Besides their usual activities, these operators are involved in some lucrative services of public interest such as mobile money (MTN and MOOV), electricity bills payment, and bank transactions but none of them provides a platform for health intervention of any kind [13]. However, they can be of help when they are associated with mobile phone-based health alerts since they already have similar platforms in other fields. The challenge of sustainability of the system due to cost and network coverage can be overcome if the system is 
TABLE 1: (a) Comparison and recapitulation of information collected from respondents. (b) Compassion and recapitulation of information collected from respondents.

(a)

\begin{tabular}{|c|c|c|c|c|}
\hline Variable & Level & Number & Proportion (\%) & $p$ value \\
\hline \multirow{2}{*}{ Phone calls } & Urban & 94 & 95.92 & \multirow{2}{*}{0.5677} \\
\hline & Rural & 32 & 100 & \\
\hline \multirow{2}{*}{ SMS } & Urban & 58 & 59.18 & \multirow{2}{*}{0.9316} \\
\hline & Rural & 18 & 56.25 & \\
\hline \multirow{2}{*}{ Digital forms } & Urban & 50 & 51.02 & \multirow{2}{*}{0.9176} \\
\hline & Rural & 16 & 50.00 & \\
\hline \multirow{2}{*}{ Phone calls } & Public & 68 & 94.44 & \multirow{2}{*}{0.1893} \\
\hline & Health professionals & 58 & 100 & \\
\hline \multirow{2}{*}{ SMS } & Public & 28 & 38.89 & \multirow{2}{*}{0.014} \\
\hline & Health professionals & 36 & 62.07 & \\
\hline \multirow{2}{*}{ Digital forms } & Public & 22 & 30.55 & \multirow{2}{*}{0.007} \\
\hline & Health professionals & 32 & 55.17 & \\
\hline \multirow{3}{*}{ Common communication methods } & SMS & 102 & 78.46 & \\
\hline & Calls & 130 & 100.00 & \\
\hline & Internet-apps & 68 & 52.31 & \\
\hline \multirow{7}{*}{ Medical use of cell phone } & Appointment & 65 & 50.00 & \\
\hline & Emergency & 15 & 11.54 & \\
\hline & Follow-up & 12 & 9.23 & \\
\hline & Complaints & 25 & 19.23 & \\
\hline & Cases & 65 & 50.00 & \\
\hline & Vaccination & 6 & 4.62 & \\
\hline & Treatments & 22 & 16.92 & \\
\hline
\end{tabular}

(b)

\begin{tabular}{|c|c|c|c|}
\hline Variable & Level & Number & Per cent (\%) \\
\hline \multirow{8}{*}{ Current disease reporting means } & TV & 92 & 70.77 \\
\hline & Newspaper & 93 & 71.54 \\
\hline & Radio & 55 & 42.31 \\
\hline & Colleagues & 24 & 18.46 \\
\hline & Internet & 78 & 60.00 \\
\hline & Relatives & 22 & 16.92 \\
\hline & Social media & 56 & 43.08 \\
\hline & Pub-shouter & 2 & 1.54 \\
\hline \multirow{14}{*}{ Reportable issues using cell phone } & Outbreak & 123 & 94.62 \\
\hline & Cases & 113 & 86.92 \\
\hline & Pregnancy & 65 & 50.00 \\
\hline & Birth & 87 & 66.92 \\
\hline & ARV-Remd & 55 & 42.31 \\
\hline & Drug-Remd & 12 & 9.23 \\
\hline & Appointment & 112 & 86.15 \\
\hline & Diagnoses & 74 & 56.92 \\
\hline & Treatment & 49 & 37.69 \\
\hline & Vaccination & 62 & 47.69 \\
\hline & Emergency & 90 & 69.23 \\
\hline & Controls & 33 & 25.38 \\
\hline & Drug purchase & 13 & 10.00 \\
\hline & Prophylaxis & 19 & 14.62 \\
\hline
\end{tabular}


supported and subsidised by the government and NGOs that advocate for public health. These partners must be associated in a nascent project in order to make the program free of charge for the public. Similarly, telecom providers will adhere to the system and support it so long as it increases their visibility and gives them more subscribers. This should be a kind of win-win partnership between telecom providers who gain in subscribers and the local and national government and NGOs who gain in achieving health related goals of their programmes. If many of these types of systems have failed in the past, it is attributable to the fact that most of them were only based on self-designed apps run independently without any prior involvement of governments and telecom providers $[6,8]$. Populations' training on the use of the tools and national sensitization are of the most important aspect to sustain the system. When the system is free of charge with good network connection, there is a compulsory need for training and capacity building in the population to improve their proficiency regarding the use of the platform in order to keep the system sustainable.

The technologies can be used to establish surveillance systems through the network of healthcare extension workers where they could be provided with a number for specific diseases needing immediate reporting in human and animals [14]. It could also be used to provide healthcare information to the general public by alerting people during emergency situations and outbreaks [14]. Such mobile technologies have the potential to revolutionize disease surveillance in developing countries especially in remote areas with difficult road infrastructure and challenging terrain. Mobile phone-based platforms can also be used for medication reminders, adherence, defaulter tracing, and animal vaccination campaign alerts. Rodrigues et al. [15] suggested that mobile phones may improve medication adherence in HIV infected individuals in Uganda. Similar utilisations were made in Kenya and SouthAfrica and proven to be very effective $[6,16]$. Furthermore, mobile phones could be used as a tool for strengthening the health management information systems (for animals and human) to facilitate collection and compilation of information from wide areas. A collaborative study conducted in Tanzania, Burundi, and Zambia concluded that mobile technology-based surveillance system is useful in providing near to real-time data, with the potential for enhancing timely response in rural remote areas of Africa [7]. There is therefore the inevitably urgent need of adopting mobile phone technologies for one health surveillance in Benin since the benefits are multiple and multiform. In a recent comparative study on mobile phone-based malaria reporting and paper based registries in Zambia, Hamainza et al. [17] concluded that health surveillance could be highly effective if mobile technology platforms are harnessed for reporting directly from community health workers to central servers to allow rapid data access, use, and quality assurance by stakeholders at local and national levels. In Nigeria, mobile phones were proven as tools for improving cancer care in low resource settings [18]. Similarly, a gynaecologic report from Ghana shows that mobile phone-based reporting from rural-based healthcare centres presents a more accurate picture of what occurs in remote communities because it happens in real time [19].
Mobile phone reporting has proven the feasibility of improving disease surveillance capacity through innovative systems despite resource constraints around the world and in Africa. In Madagascar, it was reported that a mobile phone-based surveillance system can detect unexpected increases in the incidence of influenza-like illnesses and other syndromic illnesses [20]. Likewise, a study conducted in Rwanda successfully designed and implemented a mobile phone SMS-based system to track pregnancy and maternal and child outcomes in limited resource settings [21]. A similar case was reported in Liberia [22].

The current study pointed out that for effective implementation of mobile phone-based health surveillance in Benin phone calls are more preferred than SMS and smart phone digital forms. This is constant with the most common communication methods used in the study population for routine mobile phone needs (phone calls followed by SMS and Internet applications). Nevertheless, the tendency is more pronounced among individuals from the public than healthcare professionals and could be explained by the literacy level of the population. In fact, the use of SMS and digital forms in Benin requires a user to be able to read and type in French language which is rare in a country where the national literacy level is about $42.4 \%$ [23]. Similar observations were made by Houngbo [13] who concluded in his report that despite the high teledensity of about $83.81 \%$ in Benin the use of ICTs is still low in many domains including telemedicine and e-health due to low literacy level and high internet cost. Phone calls as the most preferred method among respondents might not be practical during implementation of disease surveillance due to a number of challenges including lack of reliability, inconsistencies, and cost constraints. In previous studies which reported the use of mobile phone technologies for health interventions, SMS is the most commonly used method $[6,7,12,24]$. SMS and possibly digital forms are therefore the easiest, most reliable, and most effective methods for the implementation of mobile reporting. This is therefore an alert for any upcoming project of mobile phone-based health surveillance in Benin. Such project will have to design SMS platforms in local languages and train pilot populations on the use of the system for successful application. Besides these limitations, Haberer et al. [25] reported misunderstanding of personal identification numbers in their SMS platform as the main cause of low response rates from populations.

Although mobile phone-based technologies are still not implemented in Benin, a case study on e-surveillance in animal health was conducted in the country to monitor invasive tick species using various electronic tools such as EpiCollect, USB microscopes, and interactive, electronic Bayesian identification keys [26]. The study confirmed the feasibility of e-surveillance in the country and concluded that the evaluation of the used tools clearly shows the importance of selecting the appropriate tool depending on the envisaged data to be collected. Issues like accessibility, visualization, and cost related to data collection should therefore be taken in account while choosing a tool. 


\section{Conclusion}

The electronic disease surveillance system has improved timeliness and completeness of reporting and extremely supports early detection and notification of outbreaks for timely response. This study revealed urgent needs of mobile phone technologies for animal and human health surveillance and interventions in Benin leading to tropical disease elimination. Such systems should be rapidly adopted in Benin as the need is urgent and previous studies have confirmed its feasibility. The current findings also call for projects towards the development of mobile phone-based surveillance networks in Benin.

\section{Competing Interests}

The authors declared no competing interests with respect to the study, authorship, and publication of this article.

\section{Acknowledgments}

The authors thank the University of Abomey-Calavi in Benin, the various respondents, and the Sokoine University of Agriculture in Tanzania for their contributions to this study.

\section{References}

[1] World Health Organization, "Lassa Fever-Benin.Emergencies preparedness, response," 2016, http://www.who.int/csr/don/19february-2016-lassa-fever-benin/en/.

[2] K. E. Jones, N. G. Patel, M. A. Levy et al., "Global trends in emerging infectious diseases," Nature, vol. 451, no. 7181, pp. 990993, 2008.

[3] E. M. De Clercq, S. Leta, A. Estrada-Peña, M. Madder, S. Adehan, and S. O. Vanwambeke, "Species distribution modelling for Rhipicephalus microplus (Acari: Ixodidae) in Benin, West Africa: comparing datasets and modelling algorithms," Preventive Veterinary Medicine, vol. 118, no. 1, pp. 8-21, 2015.

[4] World Health Organization, International Health Regulations (2005), WHO, Geneva, Switzerland, 2nd edition, 2008.

[5] A. M. Kimball, M. Moore, H. M. French et al., "Regional infectious disease surveillance networks and their potential to facilitate the implementation of the international health regulations," Medical Clinics of North America, vol. 92, no. 6, pp. 1459-1471, 2008.

[6] L. Jennings, J. Ong'Ech, R. Simiyu, M. Sirengo, and S. Kassaye, "Exploring the use of mobile phone technology for the enhancement of the prevention of mother-to-child transmission of HIV program in Nyanza, Kenya: A Qualitative Study," BMC Public Health, vol. 13, no. 1, article 1131, 2013.

[7] M. Mwabukusi, E. D. Karimuribo, M. M. Rweyemamu, and E. Beda, "Mobile technologies for disease surveillance in humans and animals," Onderstepoort Journal of Veterinary Research, vol. 81, no. 2, article 737, 2014.

[8] J. Brinkel, A. Krämer, R. Krumkamp, J. May, and J. Fobil, "Mobile phone-based mHealth approaches for public health surveillance in Sub-Saharan Africa: a systematic review," International Journal of Environmental Research and Public Health, vol. 11, no. 11, pp. 11559-11582, 2014.
[9] H. Cole-Lewis and T. Kershaw, "Text messaging as a tool for behavior change in disease prevention and management," Epidemiologic Reviews, vol. 32, no. 1, pp. 56-69, 2010.

[10] Autorités de Régulations des Communications Electroniques et de la Poste (ARCEP-BENIN), Analyse de la Tendance du Secteur des Télécommunications au Bénin, ARCEP-BENIN, 2015.

[11] S. Kunutsor, J. Walley, E. Katabira et al., "Using mobile phones to improve clinic attendance amongst an antiretroviral treatment cohort in rural Uganda: a cross-sectional and prospective study," AIDS and Behavior, vol. 14, no. 6, pp. 1347-1352, 2010.

[12] D. Zurovac, R. K. Sudoi, W. S. Akhwale et al., "The effect of mobile phone text-message reminders on Kenyan health workers' adherence to malaria treatment guidelines: a cluster randomised trial," The Lancet, vol. 378, no. 9793, pp. 795-803, 2011.

[13] P. J. Houngbo, "État des lieux de l'usage des Technologies de l'Information et de la Communication au Bénin," Forum sur la Gouvernance de l'Internet au Bénin, 2015, http://researchict africa.net/ria_rap/wp-content/uploads/2015/12/E\%CC\%81tatdes-lieux-Communication-Be\%CC\%81nin.pdf.

[14] A. Little, A. Medhanyie, H. Yebyo, M. Spigt, G.-J. Dinant, and R. Blanco, "Meeting community health worker needs for maternal health care service delivery using appropriate mobile technologies in Ethiopia," PLoS ONE, vol. 8, no. 10, Article ID e77563, 2013.

[15] R. Rodrigues, A. Shet, J. Antony et al., "Supporting adherence to antiretroviral therapy with mobile phone reminders: results from a cohort in South India," PLoS ONE, vol. 7, no. 8, Article ID e40723, 2012.

[16] K. C. M. Bahadura and P. J. Murray, "Cell Phone Short Messaging Service (SMS) for HIV/AIDS in South Africa: a literature review," in MEDINFO, vol. 160 of Studies in Health Technology and Informatics, pp. 530-534, IOS Press, 2010.

[17] B. Hamainza, G. F. Killeen, M. Kamuliwo, A. Bennett, and J. O. Yukich, "Comparison of a mobile phone-based malaria reporting system with source participant register data for capturing spatial and temporal trends in epidemiological indicators of malaria transmission collected by community health workers in rural Zambia," Malaria Journal, vol. 13, article 489, 2014.

[18] V. I. Odigie, L. M. D. Yusufu, D. A. Dawotola et al., "The mobile phone as a tool in improving cancer care in Nigeria," PsychoOncology, vol. 21, no. 3, pp. 332-335, 2012.

[19] P. Andreatta, D. Debpuur, A. Danquah, and J. Perosky, "Using cell phones to collect postpartum hemorrhage outcome data in rural Ghana," International Journal of Gynecology and Obstetrics, vol. 113, no. 2, pp. 148-151, 2011.

[20] S. Rajatonirina, J.-M. Heraud, L. Randrianasolo et al., "Short message service sentinel surveillance of influenza-like illness in Madagascar, 2008-2012," Bulletin of the World Health Organization, vol. 90, no. 5, pp. 385-389, 2012.

[21] F. Ngabo, J. Nguimfack, F. Nwaigwe et al., "Designing and implementing an innovative SMS-based alert system (RapidSMS-MCH) to monitor pregnancy and reduce maternal and child deaths in Rwanda," Pan African Medical Journal, vol. 13, article 31, 2012.

[22] J. R. Lori, M. L. Munro, C. J. Boyd, and P. Andreatta, "Cell phones to collect pregnancy data from remote areas in Liberia," Journal of Nursing Scholarship, vol. 44, no. 3, pp. 294-301, 2012.

[23] Central Intelligence Agency World Factbook, Benin Demographics Profile 2014, Indexmundi, 2015, http://www.indexmundi.com/benin/demographics_profile.html. 
[24] C. Déglise, L. S. Suggs, and P. Odermatt, "SMS for disease control in developing countries: a systematic review of mobile health applications," Journal of Telemedicine and Telecare, vol. 18, no. 5, pp. 273-281, 2012.

[25] J. E. Haberer, J. Kiwanuka, D. Nansera, I. B. Wilson, and D. R. Bangsberg, "Challenges in using mobile phones for collection of antiretroviral therapy adherence data in a resource-limited setting," AIDS and Behavior, vol. 14, no. 6, pp. 1294-1301, 2010.

[26] M. Madder, J. G. Walker, J. Van Rooyen et al., "E-Surveillance in Animal Health: use and evaluation of mobile tools," Parasitology, vol. 139, no. 14, pp. 1831-1842, 2012. 


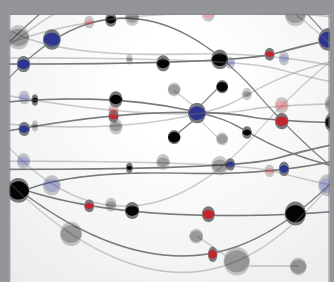

The Scientific World Journal
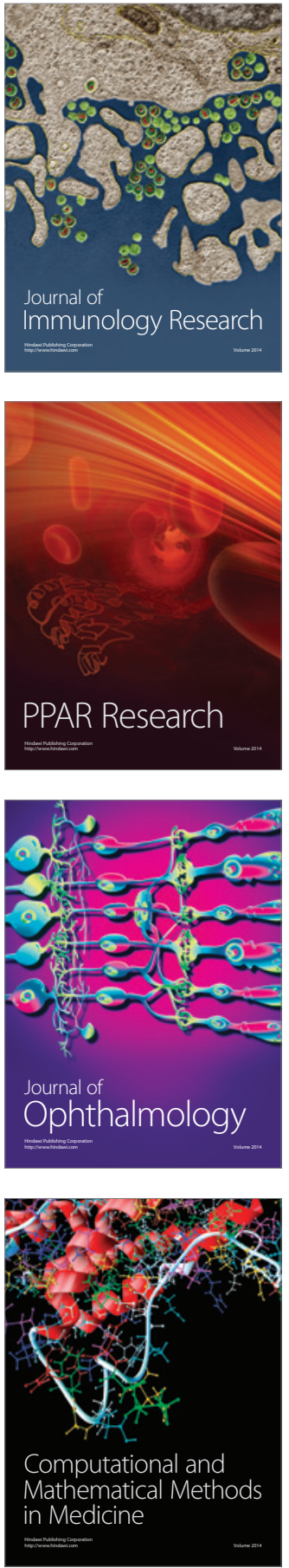

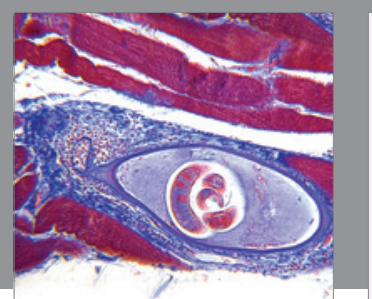

Gastroenterology Research and Practice

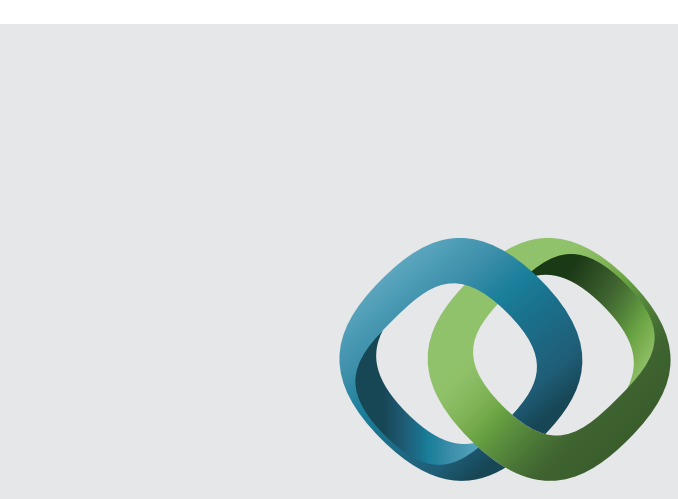

\section{Hindawi}

Submit your manuscripts at

http://www.hindawi.com
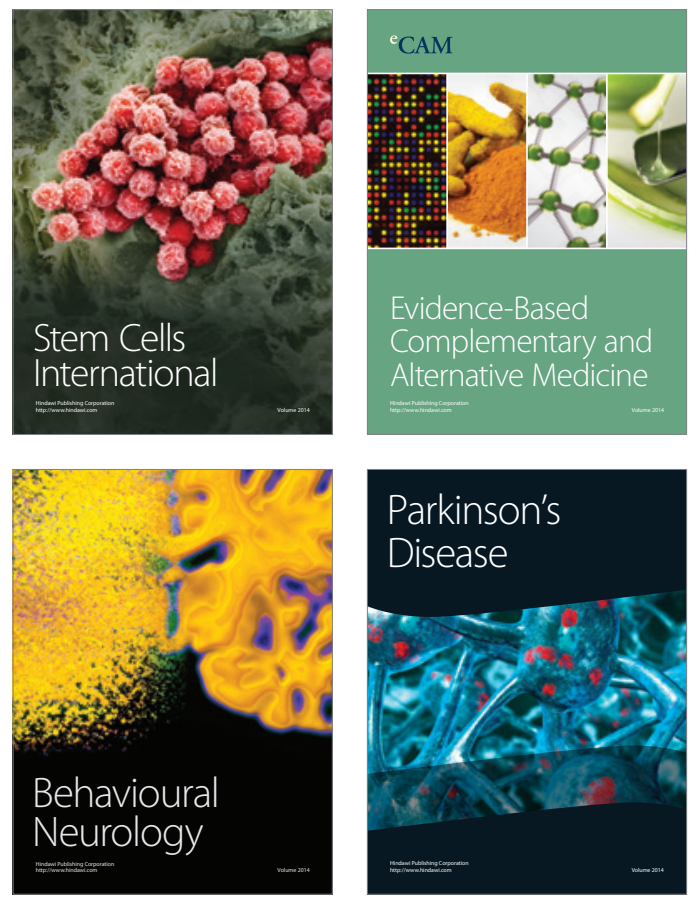
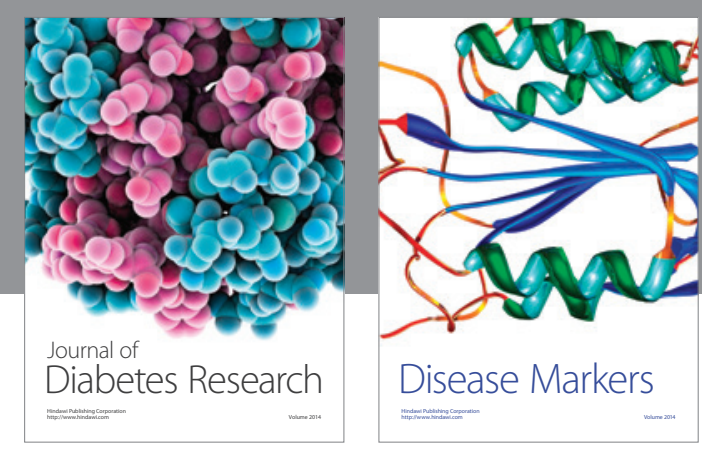

Disease Markers
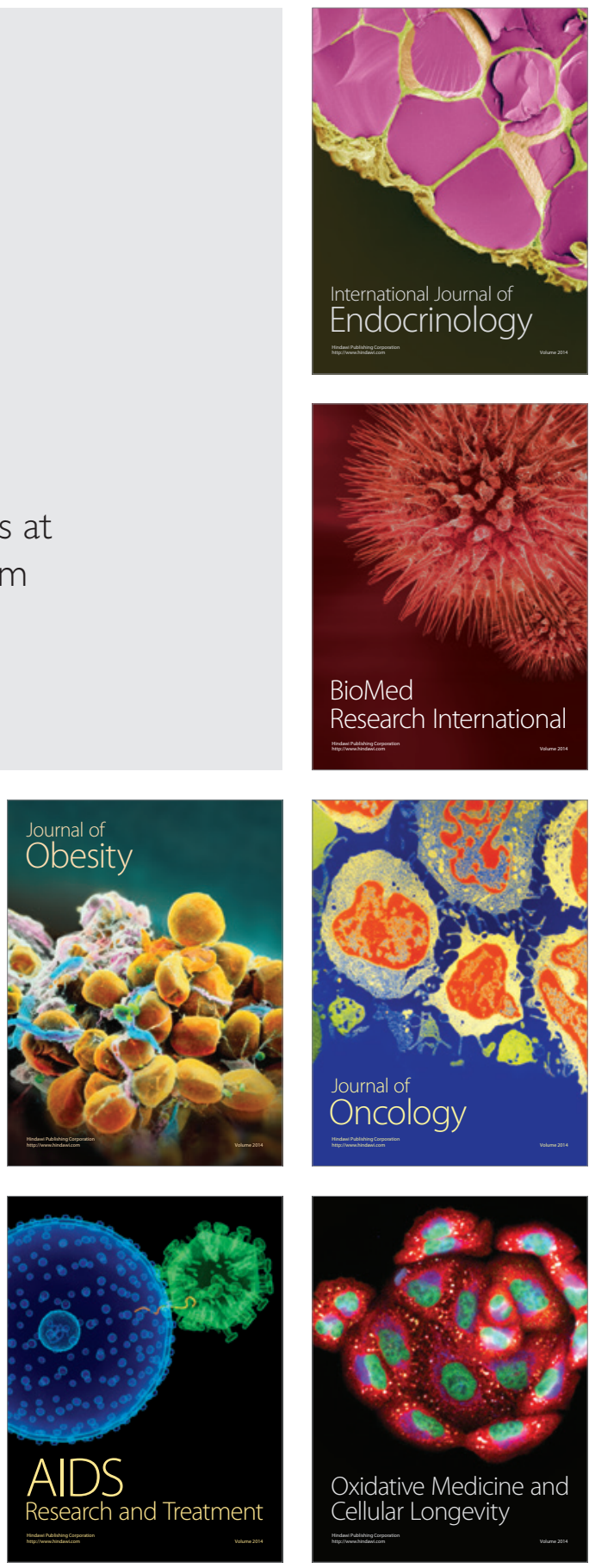